\title{
VALORACIÓN DE FUENTES RENOVABLES NO CONVENCIONALES DE GENERACIÓN DE ELECTRICIDAD: UN ENFOQUE DESDE LAS OPCIONES REALES*
}

\author{
Felipe Isaza Cuervo**
}

doi: 10.11144/Javeriana.cao28-51.vfrc. Este artículo es producto de la investigación “Evaluación de proyectos de energías alternativas mediante opciones reales y mecanismos de desarrollo limpio" financiado por la Universidad de Medellín desde marzo de 2011 hasta diciembre de 2012. El artículo se recibió el 27/08/2014 y se aprobó el 30/05/2015. Sugerencia de citación: Isaza C., F. (2015). Valoración de fuentes renovables no convencionales de generación de electricidad: un enfoque desde las opciones reales. Cuadernos de Administración, 28 (51), 45-64. http://dx.doi.org/10.11144/Javeriana.cao28-51.vfrc.

** Candidato a Doctor en Ingeniería - Industria y Organizaciones, Universidad Nacional de Colombia, Medellín, Colombia. Jefe de Ingeniería Financiera e Ingeniería Industrial de la Facultad de Ingenierías, Universidad de Medellín, Colombia.

Correo electrónico: fisaza@udem.edu.co 


\section{Valoración de fuentes renovables no convencionales de generación de electricidad: un enfoque desde las opciones reales}

\section{RESUMEN}

En este artículo se valoran las oportunidades de inversión en tecnologías emergentes de generación de electricidad, las cuales se consideran altamente riesgosas por la volatilidad del precio de mercado de la electricidad. Se utiliza el modelo propuesto por Dixit y Pindyck (1994) e información teórica de costos de inversión y operación para las tecnologías analizadas y se encuentra el valor de la oportunidad y precio óptimo de mercado para invertir en estas tecnologías en el contexto colombiano. Los resultados evidencian que para las condiciones actuales de mercado, los inversionistas requieren un precio mayor de mercado y la conveniencia de diferir la inversión, coincidiendo con el comportamiento observado en el mercado colombiano.

Palabras clave: Opciones reales, energías renovables, mercados de electricidad.

Clasificación JEL: D81, G39, Q42

\section{ABSTRACT}

In this paper the investment opportunities in emerging power generation technologies, which are considered risky investments due to the volatility of the market price of electricity, are valuated. This analysis uses both the theoretical framework for valuing real options proposed by Dixit and Pindyck (1994) and theoretical information of investment and operational costs for the technologies being analyzed. The model allows a calculation of the value of the investment opportunity and the optimal trigger price to invest in these technologies within the Colombian electricity market conditions. Results show that under current market conditions investors should defer the investment until a required optimal trigger price is found, results which also correspond to the pattern observed in the Colombian market.

Keywords: Real options, renewable energies, energy markets. JEL Classification: D81, G39, Q42

\section{Avaliação de fontes renováveis não convencionais de geração de energia: um enfoque a partir das opções reais}

\section{RESUMO}

Neste artigo, são avaliadas as oportunidades de investimento em tecnologias emergentes de geração de energia, as quais são consideradas altamente arriscadas pela volatilidade do preço de mercado da energia. Utiliza-se o modelo proposto por Dixit e Pindyck (1994), bem como informação teórica de custos de investimento e operação para as tecnologias analisadas e constata-se o valor da oportunidade e do preço ideal de mercado para investir nessas tecnologias no contexto colombiano. Os resultados evidenciam que, para as condições atuais de mercado, os investidores requerem um preço maior de mercado e a conveniência de diferir o investimento, o que coincide com o comportamento observado no mercado colombiano.

Palavras-chave: opções reais, energias renováveis, mercados de energia. Classificação JEL: D81, G39, Q42 


\section{Introducción}

Los mercados eléctricos reestructurados surgen como una respuesta a las necesidades de contar con mecanismos de negociación y de inversión en capacidad de generación eficiente frente a la creciente demanda de electricidad. Es así como a finales del siglo pasado se reestructuran los mercados de electricidad, creando sistemas de competencia regulada. Dichos mercados eléctricos abarcan la cadena de suministro de electricidad, desde la generación hasta la distribución final a los usuarios, cada una como actividades separadas y competitivas. Desde la perspectiva de la generación de electricidad, una de las principales decisiones dentro de estos mercados competitivos es la definición de la mezcla adecuada de los diferentes recursos de generación, aprovechando al máximo aquellos que están disponibles, de acuerdo con las condiciones geográficas y de recursos fósiles, en cada mercado particular. Tanto los reguladores del mercado, como los agentes generadores están expuestos a la dependencia de los recursos convencionales de generación de electricidad, siendo las centrales hidroeléctricas y térmicas (operadas con gas natural o carbón) las más tradicionales y en consecuencia son las que definen el precio de mercado de la electricidad. No obstante, las condiciones climáticas cambiantes, la regulación ambiental frente a emisiones y la posibilidad de escases y altos precios de combustibles fósiles, hacen que cada vez sea más favorable adoptar tecnologías de generación a partir de fuentes renovables no convencionales (FRNC), como por ejemplo la energía eólica, solar fotovoltaica, geotérmica y la energía hidrocinética. Si bien las FRNC presentan ventajas ambientales, algunas de sus características las convierten en inversiones riesgosas desde la perspectiva financiera, esto como consecuencia de aspectos como la intermitencia de estos recursos y su exposición a variaciones climáticas estacionales (energía eólica, solar fotovoltaica e hidrocinética), los altos costos de inversión y grado de madurez tecnológica, como la energía hidrocinética la cual se encuentra en periodos de desarrollo a gran escala. Además de lo anterior, y dadas las características particulares de cada mercado, no solo desde lo geográfico, sino también desde su matriz actual de generación y el comportamiento del precio de la electricidad, los beneficios que obtendrían los agentes generadores por la electricidad aportada por las FRNC estarían expuestas a una alta volatilidad, lo que convertiría la inversión en dichos recursos en una decisión riesgosa.

Para el caso de Colombia, país cuya generación proviene principalmente de fuentes hidroeléctricas y térmicas (gas natural y carbón), y que está expuesto a la volatilidad en precio y reservas de combustibles fósiles y a la incertidumbre sobre el fenómeno de $\mathrm{El}$ Niño, considerar la oportunidad de incorporar FRNC en la matriz de generación es prio- 
ritario (UPME, 2011). Teniendo en cuenta estos aspectos, en los últimos quince años el gobierno colombiano ha promovido a partir de leyes la inversión en FRNC, siendo la Ley 1715 de 2014 un mecanismo de fomento para la inversión en dichas alternativas a través de la propuesta de incentivos tributarios e impulso a la creación de mecanismos regulatorios que permitan incorporar a las FRNC en el mercado mayorista, participar del cargo por confiabilidad, y buscar mecanismos que desarrollen estas tecnologías en las Zonas No Interconectadas. Como consecuencia de la Ley 1715 de 2014, para las FRNC la regulación ha considerado el desarrollo de metodologías que permiten calcular la ENFICC de la energía eólica en una primera propuesta mediante la Resolución CREG 148 de 2011 la cual fue revisada y actualizada en la Resolución CREG 061 de 2015 y, más recientemente, la Resolución CREG 132 de 2014 para el cálculo de la energía firme para plantas geotérmicas; siendo las más recientes resultado de los incentivos para crear un marco regulatorio apropiado para las FRNC en Colombia como lo propone la Ley 1715 de 2014. Además, el Plan de Expansión de Generación y Transmisión 2013-2027, elaborado por la Unidad de Planeación Minero Energética (UPME, 2013) plantea la necesidad de diversificar la matriz de recursos de generación de electricidad, esto con el fin de hacer frente a la dependencia actual de grandes centrales hidroeléctricas con embalse, centrales térmicas a carbón y gas natural y contar con recursos renovables que ayuden a llevar electricidad a zonas no interconectadas. A pesar de la identificación de las necesidades, las FRNC no hacen parte de la matriz de generación colombiana, a excepción del parque eólico de Jepírachi que es el único aprovechamiento de gran escala de FRNC en Colombia; a pesar del gran potencial hidrocinético, geotérmico, eólico y solar con el que cuenta el país.

Con base en lo anterior y considerando los altos costos de inversión en estos recursos de generación y que las FRNC en Colombia no solo están expuestas a la volatilidad de los precios en el mercado de electricidad, sino también a la intermitencia del recurso (la cual disminuye su eficiencia) y la inexperiencia en el uso de estas tecnologías en el país, es importante valorar la oportunidad de invertir en estas tecnologías considerando las condiciones de riesgo e incertidumbre inherentes a este tipo de mercado y de inversiones irreversibles. Adicionalmente, aunque en la actualidad se están creando mecanismos que incentiven las FRNC para que estas se beneficien de ingresos adicionales, como el cargo por confiabilidad, aún no existen experiencias que permitan analizar los resultados de la participación de las FRNC en el mercado eléctrico colombiano.

Considerando las condiciones descritas anteriormente, en este trabajo se valora la oportunidad de inversión y el precio óptimo de mercado bajo el cual sería conveniente desarrollar inversiones en capacidad de generación mediante FRNC en el entorno del 
mercado eléctrico colombiano. Para lo anterior, se utilizará el marco teórico de valoración de opciones propuesto por Dixit y Pindyck (1994), valores teóricos de inversión y costos de operación de FRNC y las condiciones de precios de futuros en el mercado eléctrico colombiano. Mediante el marco de referencia utilizado no solo es posible valorar la oportunidad de aprovechar estos recursos en el futuro (opción de diferir), sino también explicar teóricamente por qué la adopción de estas fuentes no se ha dado aún en Colombia desde la perspectiva de que las FRNC operarían únicamente bajo condiciones de un mercado de solo energía. El supuesto de valoración bajo condiciones de un mercado de solo energía se asume, pues aunque en la actualidad se están creando mecanismos que incentiven la participación de las FRNC en el cargo por confiabilidad, aún no existe experiencia suficiente que permita analizar los resultados esperados de la participación de estas tecnologías en este mercado.

El artículo se divide en las siguiente secciones: en la primera se realiza una revisión de literatura de las opciones reales; en la segunda, se explica el modelo analítico de Dixit y Pindyck (1994) para la valoración de opciones sobre inversiones irreversibles y que es utilizado en el caso de estudio; en la tercera se analizan las variables del modelo desde la perspectiva de la valoración de inversiones en tecnologías emergentes de generación de electricidad; en la cuarta, se presenta la valoración de un caso de estudio sobre diferentes tecnologías renovables en el caso colombiano, para terminar con las conclusiones y planteamientos para desarrollos futuros.

\section{Revisión de literatura de opciones reales}

Las opciones reales fueron propuestas inicialmente por Myers (1977), quien partiendo de los trabajos de Black y Scholes (1973) y Merton (1973), identificó que las inversiones en activos reales podían ser analizadas como una analogía a las opciones financieras tipo Call. Las opciones reales son un método para valorar inversiones bajo incertidumbre y complementario a los métodos tradicionales basados en flujos de caja descontados al permitir valorar la flexibilidad de las decisiones relacionadas con la inversión en activos reales. Partiendo de los trabajos previamente mencionados se han desarrollado diversos trabajos que permiten valorar la flexibilidad de inversiones en activos reales, destacándose la propuesta de Margrabe (1978) para valorar opciones de intercambio, la valoración de inversiones con opción de abandono por Myers y Majd (1983), la valoración de la opción de diferir o aplazar la realización de la inversión (McDonald y Siegel, 1986; Pindyck, 1991); valorar la opción de intercambio de insumos o productos en una línea de producción (Kulatilaka, 1988) y las decisiones de inversión ante múltiples opciones (Trigeorgis, 1993). 
Para la valoración de opciones reales se han desarrollado diferentes modelos que se pueden agrupar en tres enfoques principales: 1) valoración mediante la solución de ecuaciones diferenciales; 2) valoración mediante mallas o árboles binomiales y 3 ) simulaciones. Los métodos que valoran mediante ecuaciones diferenciales incluye la obtención de fórmulas analíticas que son de fácil aplicación, en estos modelos se formula el comportamiento del activo subyacente a partir de ecuaciones diferenciales y restricciones que actúan como restricciones del modelo. En este grupo se destacan las propuestas de Black y Scholes (1973), Margrabe (1978), Pindyck (1991) y Dixit y Pindyck (1994). En los modelos que valoran mediante mallas binomiales se asume que el activo sigue un comportamiento binomial multiplicativo, lo cual permite representar su comportamiento de manera discreta representando diferentes escenarios durante el horizonte de evaluación. En este grupo se destacan la propuesta desarrollada por Cox, Ross y Rubinstein (1979) y Boyle (1988). Una de las principales ventajas de estos modelos es la flexibilidad para valorar opciones reales de tipo americana e incluir componentes de teoría de decisiones en la valoración (Smit y Trigeorgis, 2004). Finalmente, la valoración mediante simulación se puede considerar como una derivación de los dos métodos anteriores; la cual se utiliza principalmente cuando no se cuenta con activos réplica que permitan simular el comportamiento del subyacente o cuando existen múltiples fuentes de incertidumbre (que en algunas ocasiones no siguen los supuestos subyacentes de la valoración requeridos por los métodos anteriores); en este grupo se destacan las propuestas de Boyle (1977) y Datar y Mathews (2004).

A partir de estos trabajos, las opciones reales han sido utilizadas para valorar inversiones en sectores que están sometidos a alta incertidumbre sobre sus flujos de efectivo. En los mercados de electricidad las inversiones se realizan bajo entornos de alta incertidumbre, la cual se explica por la alta volatilidad en los precios de mercado de la electricidad y de los costos de combustibles fósiles, además de la incertidumbre con relación a algunas fuentes de generación renovable, las cuales en su mayoría dependen de condiciones climatológicas. Por lo anterior, es en los mercados de electricidad donde las opciones reales se han utilizado como herramienta de valoración y decisión para el soporte en la toma de decisiones. Entre las opciones que se han identificado en los mercados de electricidad se destacan la opción de diferir (Barria y Rudnick, 2011; Lee, 2011), opciones de crecimiento mediante construcción por etapas o modularidad (Venetsanos et al., 2002; Bednyagin y Ganansounou, 2011); flexibilidad operativa e intercambio (Marreco y Carpio, 2006; Reuter et al., 2012); expansión (Maya et al., 2012) y compuestas donde se evalúan diferentes opciones como diferir, expandir, modularidad e intercambio (Siddiqui et al., 2007; Lee y Shih, 2010) las cuales se han utilizado para valorar decisiones de inversión 
y operación, políticas energéticas y presupuestos en programas de investigación y desarrollo en tecnologías emergentes. Para mayor detalle de aplicaciones de las opciones reales en mercados de electricidad se puede consultar (Isaza y Botero, 2014).

\section{Modelo de valoración}

El marco teórico para la valoración mediante opciones reales utilizado en este trabajo está basado en las propuestas de Pindyck (1991) y Dixit y Pindyck (1994), que constituyen una extensión del modelo propuesto por McDonald y Siegel (1986) en el cual se estudia el problema del momento óptimo de la inversión en proyectos cuyo valor sigue un proceso estocástico en tiempo continuo. Bajo este marco conceptual se asume que siempre que existe una inversión irreversible hay implícita una opción de posponer o diferir el momento en el cual se toma la decisión de invertir o ejecutar el proyecto, a la vez que existe un precio crítico a partir del cual es conveniente invertir. Los principales supuestos del modelo son los siguientes:

I. El precio de mercado al cual se remuneran los bienes ofrecidos por el proyecto de inversión sigue un comportamiento browniano geométrico. Dado que el valor del proyecto es un múltiplo constante del precio de mercado, el valor del proyecto sigue un movimiento browniano geométrico que depende de los mismos parámetros que definen el comportamiento del precio (Dixit y Pindyck, 1994).

II. La variable de decisión es el valor del proyecto.

III. Es posible encontrar un precio crítico tal que se convierta en una señal de mercado para definir el momento óptimo de inversión en el proyecto.

De acuerdo con lo anterior el precio del subyacente $P$ sigue un movimiento browniano geométrico, definido como:

$$
d P=\alpha . P . d t+\sigma \cdot P \cdot d z \Rightarrow \frac{d P}{P}=\alpha . d t+\sigma . d z
$$

Donde $\sigma$ es la desviación estándar del precio del subyacente, la cual se asume constante en el tiempo; $\alpha$ es la media o parámetro de deriva que representa el comportamiento 0 variación esperada en el largo plazo en el precio del subyacente y $d z$ representa un proceso de Weiner. Suponer que el subyacente sigue un proceso browniano geométrico es importante puesto que además de representar el comportamiento estocástico del 
subyacente y del proyecto, permite obtener soluciones analíticas para la valoración del activo, tal como lo proponen Dixit y Pindyck (1994).

Un proyecto, como inversión irreversible bajo incertidumbre y sometido a las variaciones del precio del subyacente, con costo de inversión $I$, y con costos de operación $c$, donde se asume que la operación podría ser suspendida sin costos adicionales cuando el precio es inferior al costo, situación observable en los mercados eléctricos cuando los generadores regulados ofertan por encima de los costos marginales promedio con el fin de no salir despachados o para aquellos generadores inflexibles o que no están sometidos a un despacho central en el momento en el que decidan detener la generación; bajo el modelo de Dixit y Pindyck (1994) el valor de un proyecto está definido como:

$$
V(P)=\left\{\begin{array}{l}
K_{1} P^{\beta_{1}} \text { si } P<c \\
B_{2} P^{\beta_{2}}+P / \delta-c / r \text { si } P>c
\end{array}\right.
$$

Donde las constantes $K_{1}$ y $B_{2}$ son positivas y representan el valor de la opción de reanudar las operaciones en el futuro y el valor de la opción de suspensión respectivamente, y se calculan como:

$$
\begin{aligned}
& K_{1}=\frac{c^{1-\beta_{1}}}{\left(\beta_{1}-\beta_{2}\right)}\left(\frac{\beta_{2}}{r}-\frac{\beta_{2}-1}{\delta}\right) \\
& B_{2}=\frac{c^{1-\beta_{2}}}{\left(\beta_{1}-\beta_{2}\right)}\left(\frac{\beta_{1}}{r}-\frac{\beta_{1}-1}{\delta}\right)
\end{aligned}
$$

Los parámetros $\beta_{1}$ y $\beta_{2}$ se obtienen a partir de las siguientes relaciones:

$$
\begin{aligned}
& \beta_{1}=\left(\frac{1}{2}-\frac{r-\delta}{\sigma^{2}}\right)+\left\{\left[\frac{(r-\delta)}{\sigma^{2}}-\frac{1}{2}\right]^{2}+\frac{2 r}{\sigma^{2}}\right\}^{1 / 2} \\
& \beta_{2}=\left(\frac{1}{2}-\frac{r-\delta}{\sigma^{2}}\right)-\left\{\left[\frac{(r-\delta)}{\sigma^{2}}-\frac{1}{2}\right]^{2}+\frac{2 r}{\sigma^{2}}\right\}^{1 / 2}
\end{aligned}
$$


En este caso, $\delta$ es el rendimiento por conveniencia, que representa el costo de oportunidad de diferir la inversión en el proyecto pero manteniendo la opción de invertir. De acuerdo con Kudokula y Papudesu (2006), $\delta$ se puede asumir como una proporción constante de los flujos de caja esperados con relación a la inversión o valor de mercado actual del proyecto. Por su parte, $r$ representa la tasa libre de riesgo, normalmente asumida como el rendimiento promedio de los instrumentos de deuda del gobierno.

Una de las principales ventajas del modelo propuesto por Dixit y Pindyck (1994) es la posibilidad de encontrar de manera analítica un precio óptimo para el activo subyacente de manera que se obtiene una señal de mercado para tomar las decisiones de ejecución de proyectos asociados a inversiones irreversibles. El precio óptimo $\left(P^{*}\right)$ a partir del cual es conveniente ejecutar la inversión, corresponde al punto donde el valor de la opción y el valor del proyecto son tangenciales. Dicho precio se puede obtener mediante la siguiente ecuación:

$$
\left(\beta_{1}-\beta_{2}\right) B_{2}\left(P^{*}\right)^{\beta_{2}}+\left(\beta_{1}-1\right) \frac{P^{*}}{\delta}-\beta_{1}\left(\frac{c}{r}+I\right)=0
$$

La ecuación anterior se puede resolver numéricamente y además cumple que $P^{*}>c+r I$, lo que indica que el precio óptimo siempre será superior al costo de operación más el costo de capital de la inversión, cumpliendo así con los supuestos teóricos de valor agregado como requisitos para la realización de inversiones creadoras de valor.

De acuerdo con el modelo, el valor de la opción de diferir la inversión en función del precio se define como:

$$
F(P)=A_{1} P^{\beta_{1}}
$$

Donde el parámetro $A_{1}$ se obtiene una vez se conoce el precio óptimo $\left(P^{*}\right)$, dicho parámetro se encuentra mediante la siguiente expresión:

$$
A_{1}=B_{2}\left(P^{*}\right)^{\beta_{2}-\beta_{1}}+\frac{\left(P^{*}\right)^{1-\beta_{1}}}{\delta}-\left(\frac{c}{r}+I\right)\left(P^{*}\right)^{-\beta_{1}}
$$


El modelo de valoración de opciones funciona bajo el supuesto que el valor del proyecto está correlacionado directamente con los valores de mercado del producto subyacente, definidos por el precio de mercado $(P)$, y en consecuencia las variaciones que sufre este precio son replicadas por el valor del proyecto (Dixit y Pindyck, 1994).

\section{Análisis de las variables del modelo en los mercados de electricidad}

Para valorar proyectos de generación de energía eléctrica es importante analizar las características propias de la electricidad. Con el fin de poder garantizar el suministro constante y confiable de electricidad, la generación de electricidad debe ajustarse constantemente a las variaciones de la demanda, situación que se ve reflejada en la alta volatilidad de los precios de mercado o spot de la energía eléctrica; adicionalmente, dado que la electricidad no puede ser almacenada económicamente (Willems y Morbee, 2010) la energía eléctrica no se puede tomar como un commodity almacenable, por lo que no es posible considerar una unidad de electricidad como un activo financiero, eliminando la posibilidad de valoración mediante el enfoque de arbitraje utilizado en los modelos de opciones reales. Aunque una unidad de electricidad generada no se puede tener como un activo dentro de un portafolio, sí es posible hacerlo con los contratos de derivados sobre electricidad (futuros y forwards), puesto que estos permiten tener derechos, en precio, cantidad y fecha de ejercicio, que pueden ser negociados en un mercado organizado. En este sentido, mientras que la energía eléctrica generada instantáneamente y valorada al precio de mercado no es un activo financiero que pueda ser negociado en un portafolio, los contratos derivados sobre la energía generada sí lo son, y pueden ser incluidos en un portafolio que replique el comportamiento del activo subyacente. Adicionalmente, y desde la perspectiva de la valoración de proyectos, los precios de los contratos derivados reflejan los precios esperados que definen los ingresos futuros de los generadores. En la práctica muchos generadores utilizan contratos bilaterales, con precios definidos con referencia al mercado de derivados, definiendo junto con los ingresos por ventas al mercado spot el valor del proyecto.

Con base en lo anterior, para la valoración de proyectos de generación de electricidad es posible utilizar los precios de los futuros o forward sobre electricidad como precios de referencia del activo subyacente. De acuerdo con Lucia y Schwarz (2002) y Weron (2008) los precios de los futuros sobre electricidad siguen un comportamiento browniano geométrico, requerido para la aplicación del modelo de Dixit y Pindyck (1994). 
Para el mercado eléctrico colombiano, la compañía DERIVEX es la encargada de la negociación de derivados sobre productos energéticos. Los principales contratos que administra son futuros sobre electricidad mensual con vencimientos a 30, 60, 90 y 120 días, con existencia desde septiembre de 2013 de contratos con vencimiento hasta 18 meses. En este estudio se supone que los ingresos por energía vendida son almacenables como contratos financieros, así que se utilizará el precio promedio de los contratos mensuales de 90 y 120 días, los cuales permiten analizar una serie de tiempo más larga que abarca información histórica desde el comienzo del mercado de derivados. El comportamiento histórico del precio de los futuros analizados se muestra en la Figura 1.

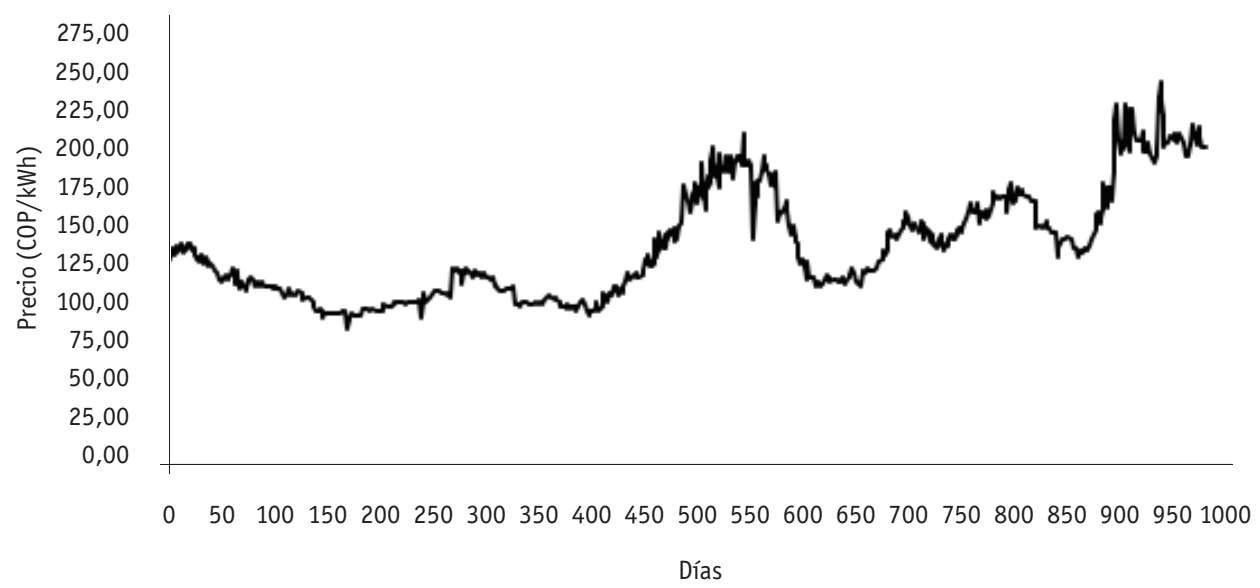

Figura 1. Precio promedio contratos de futuros (COP/kWh) sobre electricidad, 2010-2014 Fuente: elaboración propia.

De acuerdo con los supuestos del modelo descrito arriba, se asume que el precio de los futuros sigue un comportamiento browniano geométrico y el valor del proyecto se correlaciona con el precio $(P)$ de los contratos del subyacente, que para este caso se asume como el precio promedio de los contratos seleccionados, es decir, los contratos con vencimiento a 90 y 120 días. Consecuencia de lo anterior, $\sigma$ corresponde a la volatilidad de los precios futuros observados; esta se calculará sobre el retorno de la serie del precio promedio de los contratos mencionados.

Como tasa libre de riesgo $(r)$ se asume el rendimiento promedio de bonos del tesoro colombiano con vencimiento a julio de 2014, lo anterior por las características de las inversiones en activos de generación, las cuales son de largo plazo y de carácter irreversible. 
Con lo anterior, la tasa libre de riesgo se toma con un valor de 6,65\% E.A. Los valores de las variables anteriores se presentan en la Tabla 1.

\section{Tabla 1}

Parámetros iniciales de mercado para el modelo de valoración

\begin{tabular}{lc}
\hline \multicolumn{1}{c}{ Variable } & Valor \\
\hline Precio Forward $(P)$ & $\$ 137,20$ \\
Varianza $\left(\sigma^{2}\right)$ & $36,29 \%$ \\
Rendimiento Libre Riesgo $(r)$ & $6,65 \%$ \\
\hline
\end{tabular}

Fuente: elaboración propia.

El rendimiento por conveniencia $(\delta)$ se asume, de acuerdo con Kudokula y Papudesu (2006) como el costo de oportunidad de diferir el proyecto, el cual se define como una proporción de los flujos de caja esperados por la ejecución del proyecto sobre la inversión correspondiente. Los flujos de caja del proyecto se estiman utilizando valores típicos de costos y condiciones (factor de capacidad) de generación de la tecnología evaluada y los beneficios esperados por la venta de la energía eléctrica. Con el fin de estimar el rendimiento por conveniencia tal como es necesario calcular un flujo de caja anual de un proyecto típico asociado a cada tecnología evaluada, para esto se estima la generación esperada anual típica de cada tecnología, con base en el factor de capacidad y una capacidad instalada asumida, las cuales se presentan en la Tabla 2.

Además de las características propias del mercado definidas en las variables anteriores, el modelo de valoración requiere de variables específicas de inversión $(I)$ y costos de operación (c) de las tecnologías evaluadas. Una característica importante del sector eléctrico son los altos costos de inversión, lo cual constituye una barrera de entrada, especialmente para la adopción de las FRNC. Los valores estimados de los parámetros de costos de inversión y operación para cada una de las tecnologías se toman de información pública presentada por la ESMAP (2007) con proyecciones a 2015 y por OpenEI (2014). Es importante aclarar que dichos valores son generales y se utilizan principalmente para valoraciones de perfil y de competitividad de una tecnología en un mercado específico, los valores para un proyecto determinado únicamente se obtienen de un estudio detallado con las características de sitio. Tomando una tasa de cambio peso colombiano a dólar de Estados Unidos de 2.100 COP/USD, el valor de Inversión (I) y costo variable (c) se presentan en la Tabla 2. 


\section{Tabla 2}

Parámetros teóricos para la valoración de la oportunidad de inversión mediante opciones reales

\begin{tabular}{lrrrr}
\hline & \multicolumn{1}{c}{ Eólica } & Geotérmica & $\begin{array}{c}\text { Hidrocinética y } \\
\text { maremotriz }\end{array}$ & Solar fotovoltaica \\
\hline Factor de capacidad & $38 \%$ & $90 \%$ & $38 \%$ & $21 \%$ \\
Generación esperada anual [kW-h] & 166.878 .000 & 394.200 .000 & 3.337 .560 & 350.400 .000 \\
Inversión [USD/kW] & $\$ 2.000$ & $\$ 5.946$ & $\$ 6.125$ & $\$ 4.988$ \\
Capacidad instalada [kW] & 50.000 & 50.000 & 1.000 & 5.000 \\
Horizonte de evaluación & 20 & 20 & 20 & 20 \\
Costo de operación $(c)[\mathrm{COP} / \mathrm{kWh}]$ & $\$ 70$ & $\$ 80$ & $\$ 126$ & $\$ 114$ \\
Inversión unitaria $(I)[\mathrm{COP} / \mathrm{kWh}]$ & $\$ 116$ & $\$ 175$ & $\$ 506$ & $\$ 759$ \\
\hline
\end{tabular}

Fuente: elaboración propia a partir de información de ESMAP (2007) y 0penEI (2014).

La inversión unitaria anual que se muestra en la Tabla 2 representa el costo de inversión inicial por unidad de electricidad generada esperada durante la vida útil u horizonte de evaluación de la tecnología. Para esto se consideran valores típicos del Factor de Capacidad $(F C)$ que es una medida de eficiencia del recurso; para cada tecnología, la capacidad instalada definida para el análisis y el rendimiento libre de riesgo para estimar la inversión anualizada. La inversión unitaria $(I)$ se estima así:

$$
I=\frac{\text { Inversión Total.r } r /\left[1-(1+r)^{-n}\right]}{\text { Generación Esperada }}
$$

Inversión Total = Inversión por kW.Capacidad Instalada

Generación Esperada = Capacidad Instalada.FC. 8760

\section{Valoración de fuentes renovables no convencionales para el caso colombiano}

A partir de la información presentada en la sección anterior se obtienen los parámetros de valoración de la opción de diferir la inversión, ecuaciones (2) a (6), y de cálculo del precio óptimo que determina el momento adecuado para realizar la inversión: ecuación (7). Los parámetros por tipo de tecnología se muestran en la Tabla 3. 


\section{Tabla 3}

Parámetros del modelo de valoración por tipo de tecnología

\begin{tabular}{crrrr}
\hline Parámetros & \multicolumn{1}{c}{ Eólica } & Geotérmica & $\begin{array}{c}\text { Hidrocinética y } \\
\text { Maremotriz }\end{array}$ & Solar Fotovoltaica \\
\hline$\sigma^{2}$ & $36,29 \%$ & $36,29 \%$ & $36,29 \%$ & $36,29 \%$ \\
$\mathrm{R}$ & $6,65 \%$ & $6,65 \%$ & $6,65 \%$ & $6,65 \%$ \\
$\delta$ & $4,57 \%$ & $1,87 \%$ & $1,79 \%$ & $0,72 \%$ \\
$\beta_{1}$ & 1,193 & 1,077 & 1,074 & 1,029 \\
$\beta_{2}$ & $-0,307$ & $-0,340$ & $-0,341$ & $-0,356$ \\
$\mathrm{~K}_{1}$ & 7,056 & 33,436 & 34,557 & 117,789 \\
$\mathrm{~B}_{2}$ & $2.362,261$ & $3.029,781$ & $5.586,0$ & $1.225,558$ \\
\hline
\end{tabular}

Fuente: elaboración propia.

Considerando los parámetros de mercado y del modelo presentados en la Tabla 1 y en la Tabla 3, respectivamente, se estima el precio óptimo $\left(P^{*}\right)$ que determina el momento de inversión para cada una de las tecnologías evaluadas. Los resultados se presentan en la Tabla 4.

\section{Tabla 4}

Precio óptimo determinante de la inversión

\begin{tabular}{ccccc}
\hline & Eólica & Geotérmica & $\begin{array}{c}\text { Hidrocinética y } \\
\text { maremotriz }\end{array}$ & Solar fotovoltaica \\
\hline Precio óptimo $\left(P^{*}\right)[\mathrm{COP} / \mathrm{kW}-\mathrm{h}]$ & 150,4 & 183,6 & 372,2 & 223,9 \\
\hline
\end{tabular}

Fuente: elaboración propia.

El precio óptimo se interpreta como el valor mínimo de precio forward de la electricidad que incentivaría realizar la inversión; por ejemplo, para el caso de la energía eólica el precio que incentivaría invertir en la tecnología sería de alrededor de $150 \mathrm{COP} / \mathrm{kW}$ - $\mathrm{h}$; siendo este un valor piso o soporte para realizar la inversión. De manera comparativa se observa que la tecnología Hidrocinética y Maremotriz, que es la menos madura -es la expuesta a mayor incertidumbre y mayores costos de inversión- es la que tiene un mayor precio óptimo de inversión. Vale la pena señalar que a mayor rendimiento por conveniencia, existe un mayor costo de oportunidad por diferir la inversión y, en consecuencia, el precio óptimo de inversión es menor, como se puede ver para la energía eólica.

De acuerdo con la serie histórica de precios forward analizada (Figura 1), se observa que el precio forward, aunque se ha ubicado por encima del precio óptimo encontrado para 
desarrollar proyectos de energía eólica, geotérmica y solar fotovoltaica, la ocurrencia de estos valores es poco frecuente y se asocia a la volatilidad del mercado y efectos como el fenómeno de El Niño. Comparando el precio óptimo encontrado para la energía eólica con los precios históricos se observa que estos se encuentran por debajo del precio óptimo cerca del $68 \%$ del tiempo, con relación al precio óptimo de la energía geotérmica cerca del $86 \%$ del tiempo y la solar el $98 \%$. Históricamente, las energías hidrocinética y maremotriz nunca han presentado estos valores en el mercado. Lo anterior da cuenta de las razones por las cuales los inversionistas no destinan recursos para proyectos de generación mediante FRNC a pesar de que los costos de inversión se han reducido internacionalmente y ha mejorado la madurez de la tecnología.

Mediante el modelo utilizado es posible analizar el valor actual y de la opción de diferir el desarrollo de una central que explote el recurso FRNC. Teniendo como base de análisis un precio promedio de contrato de futuros de 137,2 COP/kWh, se obtienen los resultados expresados en COP/kWh que se presentan en la Tabla 5.

\section{Tabla 5}

Valor del proyecto y de la opción para un precio promedio actual de 137,2 kWh y para el precio óptimo encontrado

\begin{tabular}{lccc}
\hline \multicolumn{1}{c}{ Energía } & $\mathrm{V}(\mathrm{P})-\mathrm{I}$ & $\mathrm{F}(\mathrm{P})$ & $\mathrm{V}\left(\mathrm{P}^{*}\right)-\mathrm{I}$ \\
\hline & {$[\mathrm{COP} / \mathrm{kW}-\mathrm{h}]$} & {$[\mathrm{COP} / \mathrm{kW}-\mathrm{h}]$} & {$[\mathrm{COP} / \mathrm{kW}-\mathrm{h}]$} \\
Eólica & $\$$ & $\$$ & $\$$ \\
Geotérmica & 2.355 & 6.357 & 2.630 \\
Hidrocinética y Maremotriz & 6.513 & 6.530 & 8.937 \\
Solar Fotovoltaica & 6.304 & 18.104 & 19.129 \\
\hline
\end{tabular}

Fuente: elaboración propia.

Los resultados reflejan el comportamiento de los inversionistas frente a las FRNC. Si bien algunas de estas tecnologías pueden presentar viabilidad financiera medida desde la perspectiva de Flujos de Caja Descontados, para las condiciones actuales de mercado con las que se valora la electricidad, el valor de diferir la inversión es superior al valor de dicho proyecto, siendo la decisión más conveniente no realizar aprovechamientos inmediatos de FRNC, sino esperar hasta que las condiciones de mercado cambien y se logre sostener un piso de precios de futuros de electricidad, definido por el precio óptimo presentado en la Tabla 4. Los resultados se sirven para valorar las oportunidades no desarrolladas de generación mediante FRNC de empresas de este sector; por ejemplo si se 
cuenta con los estudios de ingeniería y derechos de explotación para una planta eólica con capacidad para generar durante 20 años 3,4 TWh, la oportunidad de dicha inversión sería de 7,8 billones de pesos colombianos. Este valor debería ser considerado e incluido en posibles negociaciones y fusiones o adquisiciones de la empresa propietaria de los estudios y derechos.

En la Figura 2 se presentan los resultados del comportamiento del valor de un proyecto eólico y el valor de diferir dicho proyecto para diferentes valores de precios de futuro de electricidad $(P)$. Gráficamente se puede observar que hasta lograr garantizar un piso definido por el precio óptimo de mercado (\$150 COP/kWh), siempre es más conveniente diferir la inversión. Vale la pena señalar que para el precio óptimo las dos gráficas son tangenciales, lo que indica la conveniencia de la ejecución de la inversión en dicho momento.

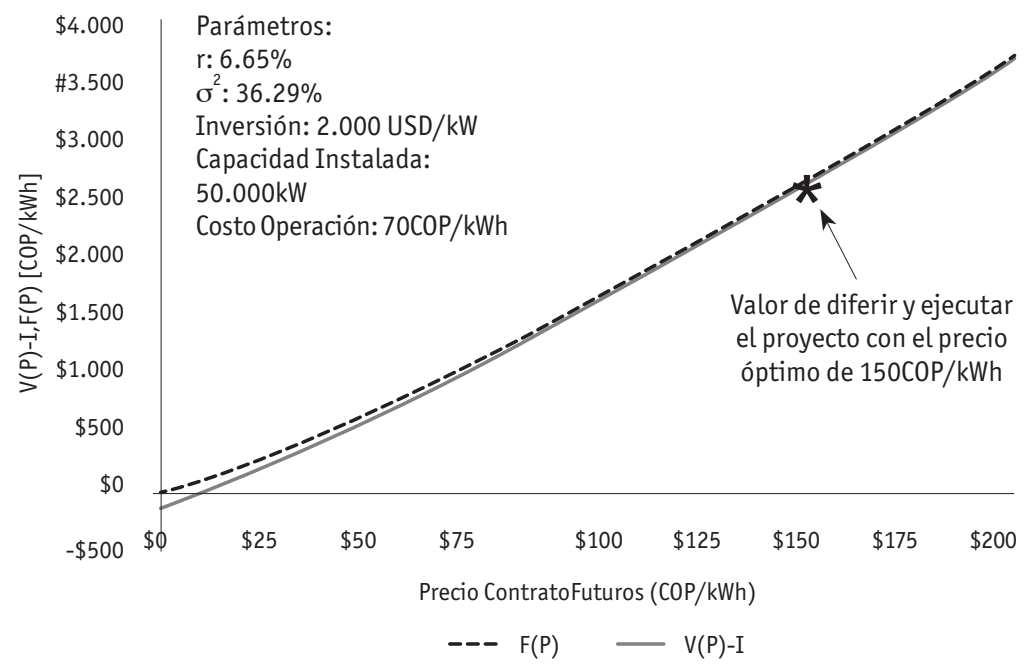

Figura 2. Valor de ejecución del proyecto $(V(P)-I)$ y de opción de diferir $(F(P))$ para diferentes precios de mercado, para Energía Eólica

Fuente: elaboración propia.

\section{Conclusiones}

En este trabajo se realiza una aplicación de valoración a través de opciones reales aplicando el marco teórico desarrollado por Dixit y Pindyck (1994). La aplicación de este modelo proporciona soluciones analíticas a la valoración de opciones permitiendo valorar no solo la inversión sino también el valor de diferir dicha opción de inversión y el precio óptimo 
que actúa como piso para la ejecución de la inversión. El modelo es aplicado para estudiar desde una perspectiva teórica y bajo el marco de las opciones reales la posibilidad de invertir en Fuentes Renovables No Convencionales (FRNC) de generación de electricidad bajo el contexto del mercado eléctrico colombiano y suponiendo que estas participan en un mercado de solo energía, sin considerar los beneficios que tendría su participación en un mercado de confiabilidad.

A partir del modelo se obtiene el precio óptimo para ejecutar una inversión que aproveche cada una de las tecnologías evaluadas. De acuerdo con los resultados se puede concluir que bajo las condiciones actuales de mercado, con precios de futuros de electricidad por debajo de los 150 COP/kWh cerca del $68 \%$ del tiempo aún se está lejos para aprovechar la opción de inversión, incluso para la tecnología renovable con las condiciones financieras más favorables, como lo es la energía eólica. Para tecnologías con poca madurez o altos costos de inversión o bajos factores de capacidad, como la energía solar fotovoltaica, hidrocinética y maremotriz, se requieren precios sostenidos de mercado que están muy lejanos de las condiciones actuales, por lo que su inclusión en la matriz de generación no es previsible en el corto plazo.

Al comparar el valor de la ejecución del proyecto $(V(P)-I)$ con el valor de la opción de diferir la oportunidad de invertir $(F(P))$ se encuentra que para las condiciones evaluadas de un precio promedio de futuro sobre electricidad de 137,2 COP/kWh, en todas las tecnologías es mayor el valor de la opción que del proyecto, en consecuencia es más conveniente diferir el desarrollo de la inversión. Este resultado es coherente con los resultados del precio óptimo de inversión y explica el rezago en la adopción de FRNC en Colombia. Adicionalmente, estos resultados confirman la necesidad de buscar mecanismos que fomenten la viabilidad financiera para incorporar estos recursos en Colombia, como por ejemplo aquellos que se derivan de lo contemplado en la Ley 1715 de 2014 con relación a beneficios tributarios y regulatorios, como aquellos que buscan diseñar mecanismos que permitan participar a las FRNC en mercados de capacidad.

Los resultados muestran que desde una perspectiva de priorización de desarrollo de tecnologías, las condiciones de mercado permiten estimar una inclusión más cercana de la energía eólica en la matriz de generación en Colombia, lo anterior por su menor precio óptimo de ejecución, el cual es muy cercano al precio promedio de mercado actual. Con relación a las tecnologías geotérmica, solar fotovoltaica y maremotriz e hidrocinética, las condiciones permiten estimar que la energía geotérmica podría tener una rápida inclusión con desarrollos de una tecnología más competitiva en términos de costos de 
inversión; mientras que para las otras dos tecnologías la inclusión en el mercado no es factible bajo las condiciones de mercado actuales; siendo posible su incorporación en sistemas por fuera del mercado o en Zonas No Interconectadas.

En este artículo también se presentó la manera como el resultado de la valoración mediante opciones puede ser utilizado para valorar los estudios y derechos de explotación de proyectos no desarrollados (diferidos) sobre FRNC de los cuales es propietaria una empresa de generación de electricidad, lo anterior con fines de valorar el negocio o realizar operaciones que impliquen la negociación de dichos derechos.

Finalmente, es importante mencionar, que aunque los resultados son coherentes con lo observado en las decisiones de inversión sobre FRNC en Colombia, los valores obtenidos son válidos para las condiciones de mercado evaluadas y los supuestos de valoración bajo condición de un mercado de solo energía, y que estos pueden cambiar por las diferentes fuentes de incertidumbre que se asocian al sector. En este caso, se consideró el efecto de la incertidumbre propia del mercado reflejada en el precio de futuros sobre electricidad, sin tener en cuenta fuentes exógenas de volatilidad como la tasa de cambio que afecta negativamente la inversión y posibles desarrollos que mejoren la eficiencia de las tecnologías evaluadas.

\section{Referencias}

Barria, C., and Rudnick, H. (2011). Investment under uncertainty in power generation: Integrated electricity prices modeling and real options approach. IEEE Latin American Transactions, 9 (5), 785-792.

Bednyagin, D., and Ganansounou, E. (2011). Real option valuation of fusion energy R\&D programme. Energy Policy, 39 (1), 116-130.

Black, F., and Scholes, M. (1973). The pricing of options and corporate liabilities. Journal of Political Economy, 81 (3), 637-654.

Boyle, P. (1977). Options: A Monte Carlo approach. The Journal of Financial Economics, 4 (3), 323-338.

Boyle, P. (1988). A lattice framework for option pricing with two state variables. The Journal of Financial and Quantitative Analysis, 23 (1), 1-12.

Cox, J. C., Ross, S. A., and Rubinstein, M. (1979). Option pricing: A simplified approach. Journal of Financial Economics, 7 (3), 229-263. 
CREG. Resolución CREG No. 061 de 2015. Por la cual se modifica la metodología para determinar la energía firme de plantas eólicas, definida mediante resolución CREG 148 de 2011 y se dictan otras disposiciones. Bogotá: Comisión de regulación de energía y gas.

CREG. Resolución CREG No. 132 de 2014. Por la cual se define la metodología para determinar la energía firme de plantas geotérmicas. Bogotá: Comisión de regulación de energía y gas.

CREG. Resolución CREG No. 148 de 2011. Por la cual se define la metodología para determinar la energía firme de plantas eólicas. Bogotá: Comisión de regulación de energía y gas.

Datar, V., and Mathews, S. (2004). European real options: An intuitive algorithm for the Black-Scholes formula. Journal of Applied Finance, 14 (1), 7-13.

Dixit, A., and Pindyck, R. S. (1994). Investment Under Uncertainty. New Jersey: Princeton University Press.

ESMAP (2007). Technical and economic assessment of off-grid, mini-grid and grid electrification technologies. Informe técnico 121/07. Recuperado en noviembre 2014, de http:// www.esmap.org

Isaza, F. y Botero, S. (2014). Aplicación de las opciones reales en la toma de decisiones en los mercados de electricidad. Estudios Gerenciales, 30 (133), 397-407.

Kudokula, P., and Papudesu, Ch. (2006). Project valuation using real options, a practitioner's guide. Fort Lauderdale: J. Ross Publishing.

Kulatilaka, N. (1988). Valuing the flexibility of flexible manufacturing systems. IEEE Transactions on Engineering Management, 35 (4), 250-257.

Lee, S. C. (2011). Using real option analysis for highly uncertain technology investments: The case of wind energy technology. Renewable and Sustainable Energy Reviews, 15 (9), 4443-4450.

Lee, S. C., and Shih, L. H. (2010). Renewable energy policy evaluation using real option model, the case of Taiwan. Energy Economics, 32 (supplement 1), S67-S68.

Lucia, J. J., and Schwartz, E. S. (2002). Electricity prices and power derivatives: Evidence from the Nordic power exchange. Review of Derivatives Research, 5 (1), 5-50.

Margrabe, W. (1978). The value of an option to exchange one asset for another. The Journal of Finance, 33 (1), 177-186.

Marreco, J. M., and Carpio, L.G.T. (2006). Flexibility valuation in the Brazilian power system: A real option approach. Energy Policy, 34, 3749-3756.

Maya, C., Hernández, J. y Gallego, 0́. (2012). La valoración de proyectos de energía eólica en Colombia bajo el enfoque de opciones reales. Cuadernos de Administración, 25 (44), 193-231.

McDonald, R., and Siegel, D. (1986). The value of waiting to invest. Quarterly Journal of Economics, 101 (4), 707-727.

Merton, R. (1973). Theory of rational option pricing. The Bell Journal of Economics and Management Science, 4 (1), 141-183. 
Myers, S. C. (1977). Determinants of corporate borrowing. Journal of Financial Economics, 5 (2), 147-175.

Myers, S. C., and Majd, S. (1983). Calculating abandonment value using option pricing theory. Working Paper 1462-83. MIT: Sloan School of Management.

Open Energy Information: OpenEI (2014). Transparent Cost Database. Recuperado en diciembre 2014, de http://en.openei.org/apps/TCDB/

Pindyck, R. S. (1991). Irreversibility, Uncertainty and Investment. Journal of Economic Literature, 29 (3), 1110-1148.

Reuter, W. H., Fuss, S., Szolgayová, J., and Obersteiner, M. (2012). Investment in wind power and pumped storage in a real options model. Renewable and Sustainable Energy Reviews, $16(4), 2242-2248$.

Siddiqui, A., Marnay, C., and Wiser, R. (2007). Real options valuation of US federal renewable research, development, demonstration and deployment. Energy Policy, 35 (1), 265-279.

Smit, H., and Trigeorgis, L. (2004). Strategic investment: Real options and games. New Jersey: Princeton University Press.

Trigeorgis, L. (1993). The nature of option interaction and the valuation of investments with multiple real options. The Journal of Financial and Quantitative Analysis, 28 (1), 1-20.

UPME (2011). Plan preliminar de expansión de referencia generación y transmisión: 2011-2025. Bogotá: Unidad de Planeación Minero Energética.

UPME (2013). Plan de expansión de referencia: generación - transmisión, 2013-2027. Bogotá: Unidad de Planeación Minero Energética.

UPME (2014). Ley 1715 de 2014. Por medio de la cual se regula la integración de las energías renovables no convencionales al sistema energético nacional. Bogotá: Unidad de Planeación Minero Energética.

Venetsanos, K., Angelopoulou, P., and Tsoutsos, T. (2002). Renewable energy sources project appraisal under uncertainty: The case of wind energy within a changing market environment. Energy Policy, 30 (4), 293-307.

Weron, R. (2008). Market price of risk implied by Asian-style electricity options and futures. Energy Economics, 30 (1), 1098-1115.

Willems, B., and Morbee, J. (2010). Market completeness: How options affect hedging and investment in the electricity sector. Energy Economics, 32, 10. 